\title{
A natural chimeric yeast containing genetic material from three species
}

\author{
Casper Groth, ${ }^{1}$ Jørgen Hansen ${ }^{2}$ and Jure Piškur ${ }^{1}$ \\ Author for correspondence: Jure Piškur . Tel: +45 4525 2518. Fax: +45 45932809. \\ e-mail: imjp@pop.dtu.dk
}

\author{
1 Department of \\ Microbiology, Technical \\ University of Denmark, \\ DTU-301, DK-2800 Lyngby, \\ Denmark \\ 2 Carlsberg Research \\ Laboratory, Gamle \\ Carlsberg Vej 10, DK-2500 \\ Valby, Denmark
}

\begin{abstract}
The Saccharomyces sp. CID1 isolate (CBS 8614) and several other Saccharomyces sensu stricto yeasts were analysed for their mitochondrial and nuclear genes. The data show that Saccharomyces Sp. CID1, found so far only in one location in Europe, is a natural hybrid between three different Saccharomyces yeast species. Two of them, Saccharomyces cerevisiae-like and Saccharomyces bayanus-like, are ubiquitous and contributed parts of the nuclear genome; the third, Saccharomyces sp. IFO 1802-like, which has been found only in Japan, contributed the mitochondrial DNA molecule. These data suggest that the yeast cell is able to accommodate, express and propagate genetic material that originates from different species, and the very existence of the resulting natural hybrids indicates that such hybrids are well adapted to their habitats.
\end{abstract}

Keywords: yeast, taxonomy, hybridization, mitochondrial DNA, MET2

\section{INTRODUCTION}

Horizontal transfer of genetic material is not as frequent among eukaryotes as it is among bacteria. However, hybridization between two species occurs occasionally in nature, and among plants the resulting hybrids are sometimes viable and can propagate. So far, there is only limited knowledge about horizontal transfer and resulting hybrids among yeasts and other fungi.

The best-described example of a fungal hybrid is the lager brewing yeast, Saccharomyces pastorianus (synonym Saccharomyces carlsbergensis). This cultured yeast is an allotetraploid hybrid between two yeast species belonging to the Saccharomyces sensu stricto group, the baker's yeast, Saccharomyces cerevisiae, and an unknown, Saccharomyces bayanus-related yeast. One possibility is $S$. bayanus CBS 1503 (syn. Saccharomyces monacensis). Almost complete parental chromosome sets are preserved in $S$. pastorianus (Hansen \& Kielland-Brandt, 1994; Kielland-Brandt et al., 1995; Pedersen, 1986), while the mitochondrial

Abbreviation: mtDNA, mitochondrial DNA.

The GenBank/EMBL accession numbers for the nuclear MET2 and mitochondrial ATP8, ATP9 and SSU gene sequences reported in this study are AF112009, AF112010, AF114899, AF114901-AF114906 AF114908, AF114909, AF114911-AF114916, AF114918-AF114920, AF114922AF114924, AF114927, AF114929, AF114930 and AF114933. genome (mtDNA) originates from the non-S. cerevisiae parent (Piškur et al., 1998). Recently, several novel yeast isolates were analysed for the structure of their nuclear and mitochondrial genomes. When a cider yeast isolate from a home brewery in Brittany, Saccharomyces sp. CID1 (CBS 8614), was analysed, some peculiar features of its origin became apparent.

Saccharomyces sp. CID1 was shown by nucleotide sequencing to contain two versions of the nuclear $M E T 2$ gene, an $S$. cerevisiae-like allele and an $S$. bayanus-like allele. Furthermore, karyotyping revealed $S$. cerevisiae-like as well as $S$. bayanus-like chromosomes (Masneuf et al., 1998). However, sequencing of the mitochondrial $A T P 9$ gene revealed that the Saccharomyces sp. CID1 allele diverged equally from those of the type strains of $S$. cerevisiae and $S$. bayanus (Masneuf et al., 1998). In this report, screening of several different isolates of the Saccharomyces sensu stricto group is described in order to find the origin of Saccharomyces sp. CID1.

\section{METHODS}

Yeast strains. The yeast strains used in this study were: $S$. bayanus CBS $380^{\mathrm{T}}$, S. bayanus CBS 395 (syn. Saccharomyces uvarum), S. pastorianus CBS $1538^{\mathrm{T}}, S$. pastorianus CBS 1513 (syn. S. carlsbergensis), S. pastorianus CBS 1503 (syn. S. monacensis), Saccharomyces paradoxus NRRL Y-1721 7 ${ }^{\mathrm{T}}, S$. paradoxus CBS 2908 (syn. Saccharomyces douglasii), Saccharomyces sp. CID1 (CBS 8614) and Saccharomyces sp. 
Table 1. Primers used for sequencing the mitochondrial small rRNA gene $S S U$ and the mitochondrial ATP synthase subunit 8 and 9 genes ATP8 and ATP9

\begin{tabular}{|c|c|}
\hline Primer & Sequence \\
\hline \multicolumn{2}{|l|}{$S S U$} \\
\hline SRNA YM-5 (forward) & 5'-AAGAATATGATGTTGGTTCAGA \\
\hline SRNA YM-9 (forward) & 5'-CAGCAGTGAGGAATATTGCAGAAT \\
\hline SRNA YM-8 (forward) & 5'-TGGTTTAAAGGATCCGTAGAAT \\
\hline SRNA YM-10 (forward) & 5'-GACGGTTACAGACTTAAGCAGTG \\
\hline SRNA YM-11 (forward) & 5'-CTAGAGTAGCGAAACGGATTCG \\
\hline SRNA YM-12 (forward) & 5'-GTTGTCTTTAGTTCGTGCTG \\
\hline SRNA YM-14 (forward) & 5'-CGGTGAATATTCTAACTGTTTCGC \\
\hline SRNA YM-16 (forward) & 5'-TACAGTTACCGTAGGGGAACCTGC \\
\hline SRNA YM-6 (reverse) & 5'-TCTGAACCAACATCATATTCTT \\
\hline SRNA YM-7 (reverse) & 5'-CAATATTCCTCACTGCTGTATCTTATAG \\
\hline SRNA YM-13 (reverse) & 5'-ATTCTACGGATCCTTTAAACCA \\
\hline SRNA YM-15 (reverse) & 5'-CGAATCCGTTTCGCTACTCTAG \\
\hline SRNA YM-17 (reverse) & 5'-CAGCACGAACTAAAGACAAC \\
\hline SRNA YM-20 (reverse) & 5'-AGGATCATTATGATTTGTCTTAATTC \\
\hline SRNA YM-18 (reverse) & 5'-GCGAAACAGTTAGAATATTCACCG \\
\hline SRNA YM-19 (reverse) & 5'-GCAGGTTCCCCTACGGTAACTGTA \\
\hline \multicolumn{2}{|l|}{ ATP8 } \\
\hline AAP1 YM-1 (forward) & 5'-ATGCCACAATTAGTTCCATTTTA \\
\hline AAP1 YM-2 (reverse) & 5'-TAATTTAGAAATAAATAATCTAGATAC \\
\hline \multicolumn{2}{|l|}{$A T P 9$} \\
\hline OLI1 YM-1 (forward) & 5'-GCAATTAGTATTAGCAGCTAAATATATTGG \\
\hline OLI1 YM-4 (reverse) & 5'-AATAAGAATGAAACCATTAAACAGA \\
\hline
\end{tabular}

IFO 1802. The strains carrying the CBS designation were obtained from the Centraal Bureau voor Schimmelcultures, Delft, The Netherlands. Strain NRRL Y-17217 ${ }^{\mathrm{T}}$ originated from the National Center for Agricultural Utilization Research, Peoria, IL, USA. Strain IFO 1802 was obtained from the culture collection of the Institute of Fermentation, Osaka, Japan. CID1 is a cider yeast, isolated from a mixed culture, collected from the bottom of a bottle of home-made apple cider from Brittany, France.

Preparation and sequencing of MET2 gene fragments. MET2 fragments from Saccharomyces sp. IFO 1802 and $S$. paradoxus CBS 2908 were amplified by PCR using primers 5'CGGCTCTAGACGAAAACGCTCCAAGAGCTGG-3' and 5'-CGGCTCTAGAGACCACGATATG CACCAGGCAG-3', which possess terminal $X b a I$ restriction sites in addition to four arbitrary bases, thus allowing restriction digestion. Genomic DNA was prepared from liquid yeast cultures (Hoffman \& Winston, 1987). For each DNA preparation, $10 \mu \mathrm{l}$ of a 100 -fold dilution was used as PCR template. The PCR was performed on a Stratagene Robocycler 40 for 25 cycles of $1 \mathrm{~min}$ at $94{ }^{\circ} \mathrm{C}, 2 \mathrm{~min}$ at $50^{\circ} \mathrm{C}$ and $3 \mathrm{~min}$ at $72{ }^{\circ} \mathrm{C}$, followed by $72^{\circ} \mathrm{C}$ for $10 \mathrm{~min}$ (one cycle). Eight independent reactions with DNA template from each isolate were performed. Each series of identical reactions was pooled and the amplified DNA was precipitated, washed and redissolved in an appropriate volume of water before being used for direct sequencing or cloning. DNA fragments were isolated from agarose by using Bio-Rad Prep-A-Gene purification matrix. The sequencing reactions were performed on a Perkin Elmer DNA Thermocycler 480 and sequences were run on an Applied Biosystems Sequenator 310. Primers for direct sequencing were identical to those used for PCR amplification, except that no restriction sites or additional arbitrary bases were included (Hansen \& Kielland-Brandt, 1994). Sequencing of the cloned fragments was performed employing the same primers or standard M13 primers. Both strands of the DNA were sequenced in all cases.

Cloning of MET2 DNA fragments. PCR-amplified MET2 fragments were cloned into pUC19 as follows. Precipitated, redissolved DNA was restricted with $X b a \mathrm{I}$ and the resulting DNA fragments were purified from agarose using Bio-Rad Prep-A-Gene purification matrix. These fragments were ligated into pUC19 vectors that had been linearized with $X b a \mathrm{I}$ and treated with calf intestine alkaline phosphatase. The resulting plasmids used for sequencing were: pJH192, pJH193 and pJH194, containing IFO 1802 MET2 inserts, and pJH195, pJH196 and pJH197, containing CBS 2908 $M E T 2$ inserts.

Isolation and sequencing of mtDNA. For isolation of DNA, a pre-culture was grown overnight in YPD medium $(2 \%$ glucose, $0.5 \%$ yeast extract, $1.0 \%$ peptone) and then the culture was grown overnight in GlyYP ( $2 \%$ glycerol, $0.5 \%$ yeast extract, $1.0 \%$ peptone) at $25^{\circ} \mathrm{C}$. Spheroplasts were prepared using zymolyase and lysed with SDS. mtDNA was separated from the other DNA using a bisbenzamide $/ \mathrm{CsCl}$ gradient (Piškur, 1989).

The sequences of the mitochondrial $A T P 8, A T P 9$ and $S S U$ genes were obtained by direct sequencing of purified mtDNA (Groth, 1998). The primers used are listed in Table 1.

Sequence analysis. The partial nuclear $M E T 2$ and the mitochondrial $A T P 8, A T P 9$ and $S S U$ gene sequences were aligned using the multiple-sequence alignment program 
Table 2. Accession numbers of the nuclear MET2 and mitochondrial ATP8, ATP9 and SSU gene sequences

Sequences have been deposited in GenBank or EMBL. The $M E T 2$ sequence from $S$. pastorianus, accession number L16688, originates from S. pastorianus production strain M204 (Hansen \& Kielland-Brandt, 1994). The $A T P 9$ and MET2 sequences from S. bayanus CBS 380 and Saccharomyces sp. CID1 are from Masneuf et al. (1998). The ATP9 sequence from S. bayanus CBS 380 and Saccharomyces sp. CID1 were deposited in EMBL. ND, Not determined

\begin{tabular}{|c|c|c|c|c|}
\hline Strain & ATP8 & ATP9 & $S S U$ & MET2 \\
\hline S. bayanus CBS $380^{\mathrm{T}}$ & AF114899 & Y16965 & AF114901 & AF112004 \\
\hline S. bayanus CBS 395 & AF114929 & $\mathrm{AF} 114930$ & AF114933 & ND \\
\hline S. pastorianus CBS $1538^{\mathrm{T}}$ & AF114923 & AF114924 & AF114927 & ND \\
\hline S. pastorianus CBS 1513 & AF114903 & AF114902 & AF114904 & ND \\
\hline S. pastorianus CBS 1503 & AF114915 & AF114916 & AF114918 & ND \\
\hline S. paradoxus NRRL Y-17217 & AF114919 & AF114920 & AF114922 & ND \\
\hline S. paradoxus CBS 2908 & AF114909 & AF114908 & AF114911 & AF112009 \\
\hline CID1 (CBS 8614) & AF114905 & Y16964 & AF114906 & $\begin{array}{l}\text { AF112005, } \\
\text { AF112006 }\end{array}$ \\
\hline IFO 1802 & AF114912 & AF114913 & AF114914 & AF112010 \\
\hline
\end{tabular}

CLUSTAL w (Thompson et al., 1994). Phylogenetic analyses were performed by using the PHYLIP phylogeny inference package (Felsenstein, 1989). Distance matrices were obtained by using the DNADIST program and unrooted phylogenetic trees were constructed using the unweighted pair group method with averages (UPGMA) and the NEIGHBOR program. Subsequently, the trees were rooted and displayed by using the tree-drawing program TREEVIEW (Page, 1996). The stability of individual branches was assessed by using the bootstrap method with the SEQBOOT, DNADIST, NEIGHBOR and CONSENSE programs of the PHYLIP package. The nuclear $M E T 2$ and mitochondrial $A T P 8, A T P 9$ and $S S U$ gene sequences that were determined in this study were deposited under the GenBank accession numbers shown in Table 2.

\section{RESULTS AND DISCUSSION}

\section{Comparison of mitochondrial genes from several yeasts}

The Saccharomyces sensu stricto group represents a closely related biological species complex that includes the currently recognized yeast species $S$. bayanus, $S$. cerevisiae, $S$. paradoxus and $S$. pastorianus, a number of subspecies and several new isolates that may represent new species (Masneuf et al., 1998; Naumov, 1996; Naumov et al., 1995a, b). The mitochondrial $A T P 8, A T P 9$ and $S S U$ genes have been sequenced from several of these yeasts. Note that the currently recognized type strains and their synonymous isolates showed identical or almost identical sequences in the coding and noncoding regions of the three mitochondrial genes analysed (data are accessible through the sequences deposited with GenBank as listed in Table 2; only the type strains are shown in Figs 1-4), thus confirming the presently accepted taxonomy of the genus (Barnett, 1992).

The ATP8 gene is the shortest mitochondrial gene. In $S$. cerevisiae, the open reading frame consists of $144 \mathrm{bp}$,

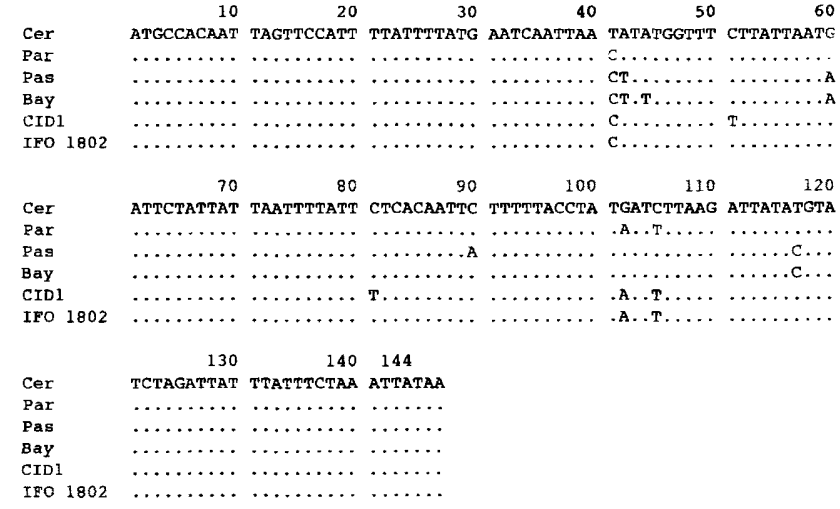

Fig. 1. The open reading frames of the mitochondrial ATP8 genes from S. bayanus (Bay), S. cerevisiae (Cer) (Macreadie et al., 1983), S. paradoxus (Par), S. pastorianus (Pas), IFO 1802 and CID1. The sequences begin with the start codon, ATG, and finish with the stop codon, TAA. Identical nucleotides are indicated by dots.

which corresponds to 48 amino acids (Macreadie et al., 1983). The ATP8 open reading frames in the yeasts examined were also 144 bp long (Fig. 1). Nucleotide substitutions were predominantly silent, with only three mutations leading to changes in the amino acid sequence. The mitochondrial ATP 8 gene sequence in Saccharomyces sp. IFO 1802 was identical to that from $S$. paradoxus, whereas the sequences of other $S a c$ charomyces species, including Saccharomyces sp. CID1, were different (Fig. 1).

The $A T P 9$ gene is larger than $A T P 8$ and is one of the most conserved mitochondrial genes. In $S$. cerevisiae and $S$. paradoxus (syn. $S$. douglasii), the open reading frame consists of $228 \mathrm{bp}$, which corresponds to 76 amino acids. Only three silent substitutions were found 


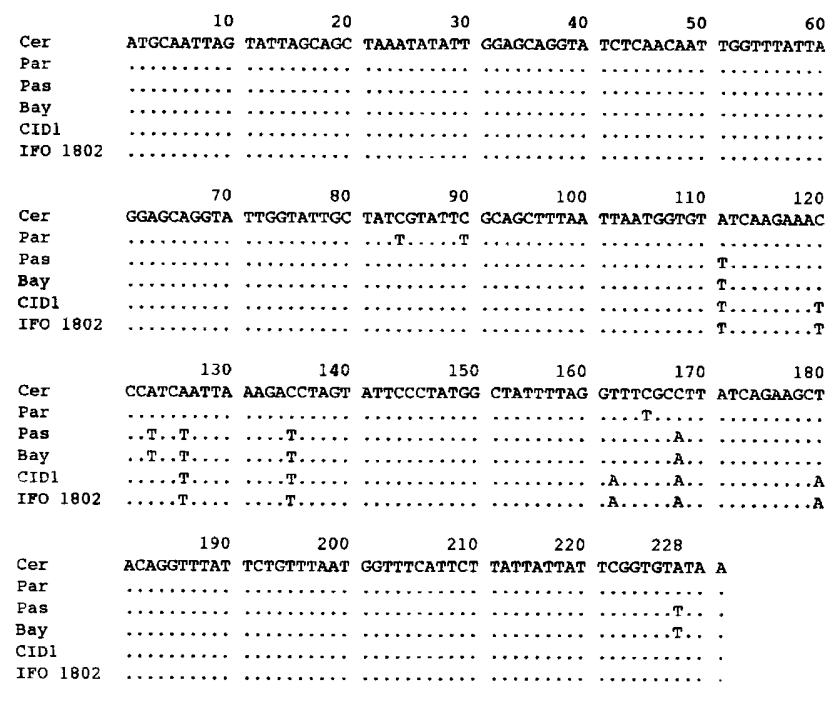

Fig. 2. The open reading frames of the mitochondrial ATP9 genes from S. bayanus (Bay) (Masneuf et al., 1998), S. cerevisiae (Cer) (Ooi et al., 1985), S. paradoxus (Par) (this study), S. pastorianus (Pas) (Masneuf et al., 1998), IFO 1802 (this study) and CID1 (Masneuf et al., 1998). The sequences begin with the start codon, ATG, and finish with the stop codon, TAA. Identical nucleotides are indicated by dots.

between these two species (Nicoletti et al., 1994; Ooi et al., 1985). The ATP9 genes in the isolates analysed were also found to be 228 bp long (Fig. 2). The amino acid sequences were identical in all cases, but several silent substitutions were observed. The ATP9 gene sequences of Saccharomyces sp. CID1 and Saccharomyces sp. IFO 1802 were identical. Also, the ATP9 sequences from $S$. pastorianus and $S$. bayanus were identical, while the sequences of other Saccharomyces species were different (Fig. 2). The data on the coding regions of the $A T P 8$ and $A T P 9$ genes suggested that a likely donor of the CID1 mitochondrial genome could be found among $S$. paradoxus-like and/or Saccharomyces sp. IFO 1802-like yeasts. However, the degree of polymorphy within these two genes was too low to obtain a more precise answer. To confirm further the origin of the CID1 mtDNA, the mitochondrial $S S U$ gene was sequenced from several yeasts.

The complete $S S U$ gene sequences each consisted of approximately 1600 nucleotides. The $S S U$ sequence of Saccharomyces sp. CID1 was shown to be clearly divergent from S. cerevisiae and S. bayanus, but $99 \cdot 4 \%$ identical to Saccharomyces sp. IFO 1802. Other species of the Saccharomyces sensu stricto complex showed 93.8-95.3\% identity (Fig. 3). The $S S U$ gene from $S$. pastorianus showed $94.5 \%$ sequence identity to $S$. cerevisiae $S S U$ and $98.4 \%$ identity to $S$. bayanus $S S U$ (Fig. 3), confirming the $S$. bayanus-like origin of its mitochondrial molecule. The number of data and the extent of polymorphy within the $S S U$ gene were adequate to establish a rather precise phylogenetic tree. These results show that the mitochondrial genomes of Saccharomyces spp. CID1 and IFO 1802

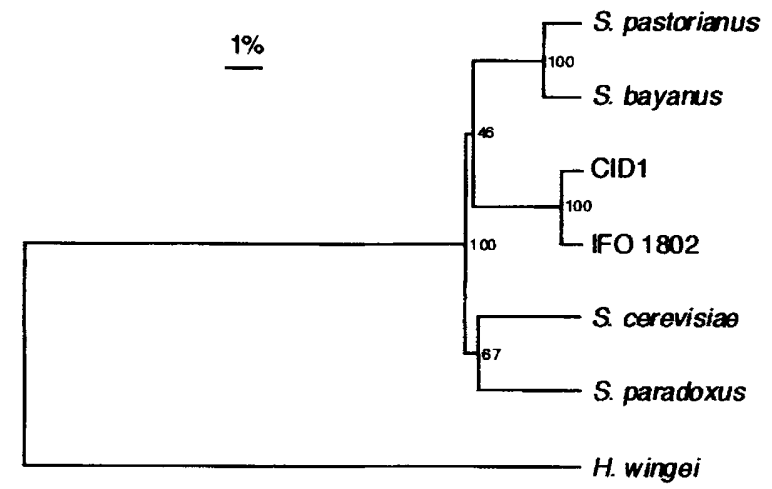

Fig. 3. Phylogenetic tree based on the mitochondrial SSU genes from S. bayanus, S. cerevisiae (Li et al., 1982), S. paradoxus, S. pastorianus, IFO 1802 and CID1. Note that the sequences can be obtained elsewhere (Table 2) and are not shown in this paper because of their extent. The bar represents $1 \%$ difference between sequences. Hansenula wingei was used as an outgroup (Sekito et al., 1995). The stability of branches is represented by percentage bootstrap values (100 cycles were performed).

are closely related. However, this relationship could be interpreted in two ways: Saccharomyces sp. IFO 1802 may be a CID1-like hybrid, or may be the parental donor of the mitochondrial genome to the CID1 hybrid.

\section{Nuclear genome of IFO 1802}

Saccharomyces sp. IFO 1802 (Kaneko \& Banno, 1991) was identified as a genetically isolated population of the Saccharomyces sensu stricto yeasts (Naumov et al., 1995b). The nature of Saccharomyces sp. IFO 1802 was investigated by analysis of nuclear DNA at the $M E T 2$ locus (Fig. 4). Note that CID1 contains two different MET2 alleles (Masneuf et al., 1998). RFLP analysis of IFO 1802 MET2 PCR fragments indicated that this isolate only contained one MET2 allele (data not shown) and thus it is not likely that this yeast is a hybrid. This finding was supported by Southern hybridization of genomic IFO 1802 DNA digested with six different restriction enzymes and probed with IFO 1802 MET2. Invariably, only one distinct signal was found (data not shown). The MET2 DNA sequence of the IFO 1802 yeast was then determined. The nucleotide sequence of the $330 \mathrm{bp}$ central region of the amplified IFO 1802 MET2 DNA fragment was obtained through sequencing of the MET2 PCR product, either by direct sequencing or by sequencing of subcloned PCR fragments in the plasmids pJH192, pJH193 and pJH194. This provided unambiguous sequences from the Saccharomyces sp. IFO 1802 $M E T 2$. The $S$. paradoxus CBS 2908 MET2 region was also determined by sequencing the inserts of $\mathrm{pJH} 195$, pJH196 and pJH197.

The $M E T 2$ allele of IFO 1802 varied from all previously sequenced Saccharomyces MET2 alleles 
(a)

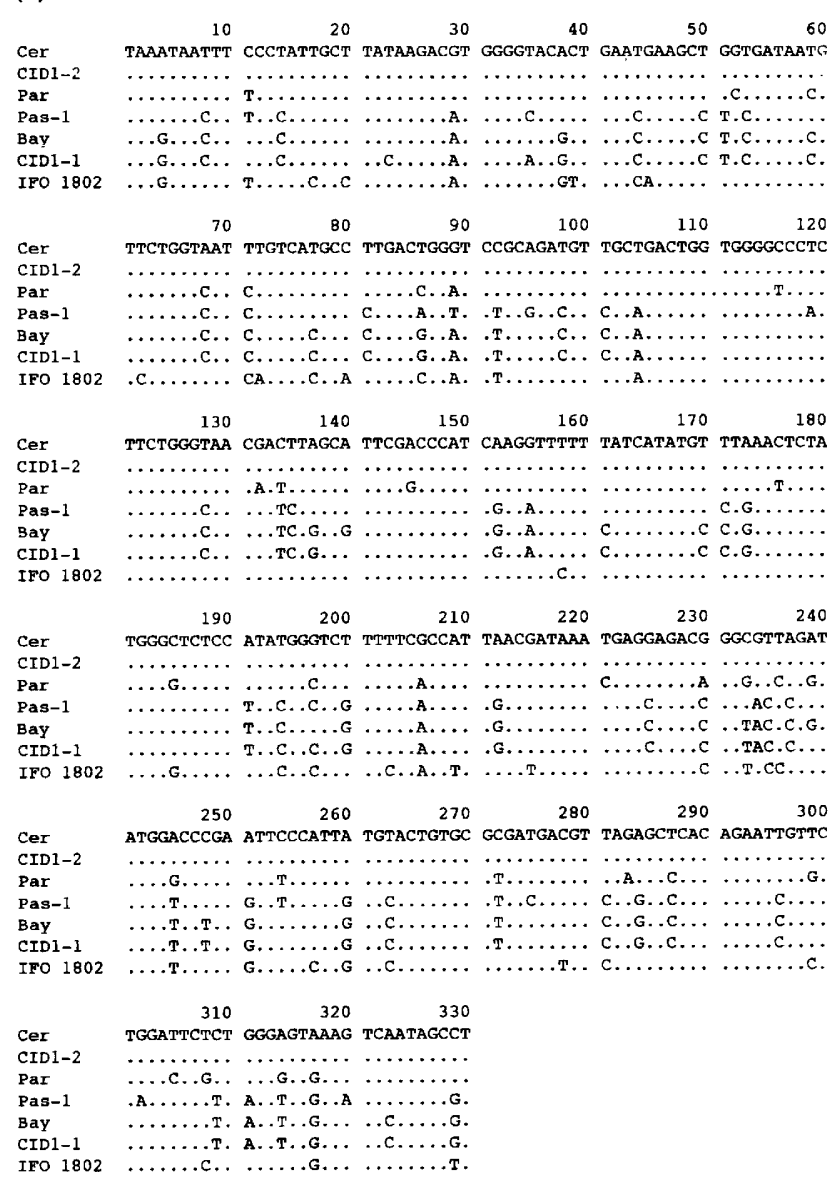

(b)

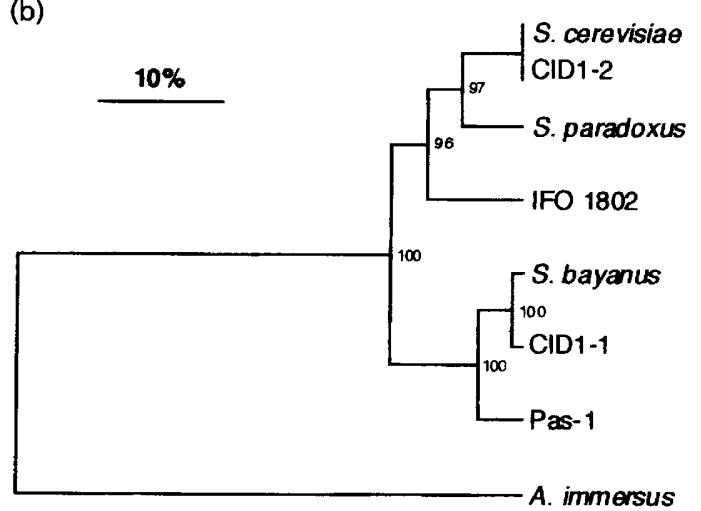

Fig. 4. Partial nucleotide sequences of the MET2 genes (a) and a phylogenetic tree based on these sequences (b) for $S$. bayanus (Bay) (Hansen \& Kielland-Brandt, 1994), S. cerevisiae S288 (Cer) (Langin et al., 1986), S. paradoxus (Par) (this study), IFO 1802 (this study), the $S$. bayanus-like allele from CID1 (CID1-1) (Masneuf et al., 1998) and S. pastorianus production strain M204 (Pas-1) (Hansen \& Kielland-Brandt, 1994) and the $S$. cerevisiae-like allele from CID1 (CID1-2) (Masneuf et al., 1998). In (a), dots denote identity to the $S$. cerevisiae MET2 sequence. In (b), the bar represents $10 \%$ difference between sequences. Ascobolus immersus was used as an outgroup (Goyon et al., 1988 ) and percentage bootstrap values are shown at individual branches (100 cycles were performed).

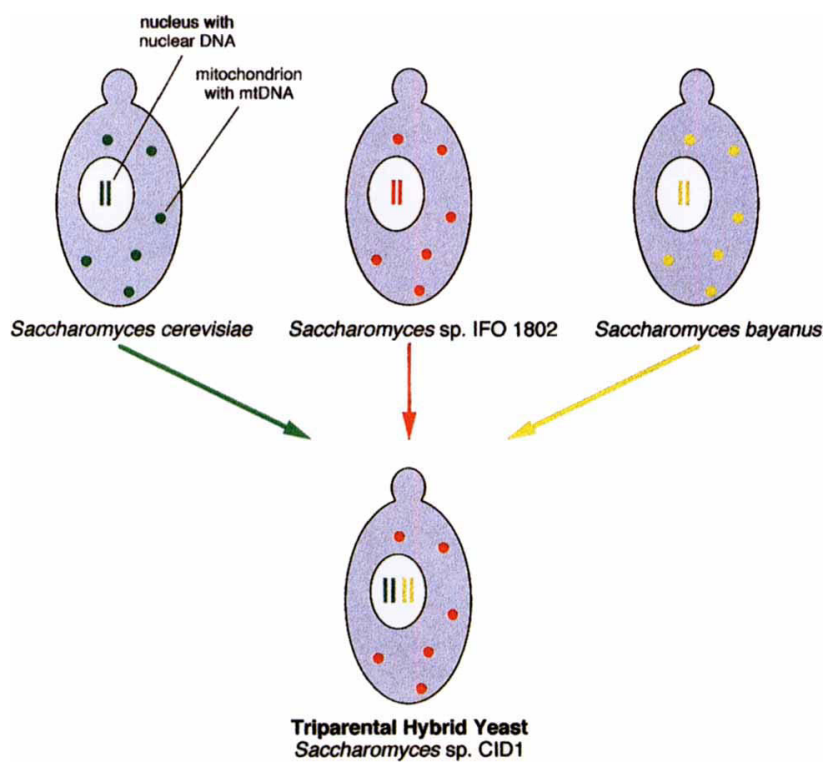

Fig. 5. The origin of a yeast with three parents. S. bayanus-like and $S$. cerevisiae-like yeasts contributed to the nuclear genome and a Saccharomyces sp. IFO 1802-like yeast contributed the mitochondrial genome to the triple hybrid yeast Saccharomyces sp. CID1 (CBS 8614).

(Hansen \& Kielland-Brandt, 1994; Langin et al., 1986; Masneuf et al., 1998) as well as from MET2 of $S$. paradoxus. The nucleotide sequence shows $88 \%$ identity to $S$. cerevisiae $M E T 2,85 \%$ identity to $S$. paradoxus $M E T 2$ and $82 \%$ identity to the S. bayanus MET2 and the $S$. bayanus-like MET2 allele of the lager brewing yeast, and $83 \%$ identity to the $S$. bayanus-like $M E T 2$ allele of the Saccharomyces sp. CID1 hybrid (Fig. 4). These data show that Saccharomyces sp. IFO 1802 is a distinct species belonging to the Saccharomyces sensu stricto complex.

\section{Origin of Saccharomyces sp. CID1}

Our data suggest that Saccharomyces sp. CID1, found so far only in one location in Europe, is a hybrid between three different Saccharomyces yeasts. Two of them, S. cerevisiae-like and S. bayanus-like, are ubiquitous and contributed parts of the nuclear genome (Masneuf et al., 1998); the third, a Saccharomyces sp. IFO 1802-like yeast, which has been found only in Japan, contributed the mtDNA molecule (Fig. 5). However, the Saccharomyces sp. IFO 1802-like ancestor could also have contributed some of the nuclear genome of Saccharomyces sp. CID1. It is difficult to determine the nature of single steps in the origin of this triple hybrid and the geographical location of each event. However, it is likely that at least two interspecific mating events occurred and that parts of the parental genetic material, specifically mtDNA from two parents and the nuclear genome from a third parent, were lost from intermediate hybrids before the final and genetically stable triple hybrid arose. 
Our results show that the fungal cell is able to accommodate, express and propagate genetic material that originates from different species, and the very existence of the resulting hybrids indicates that they are well adapted to their habitats. In this respect, fungi resemble members of the plant kingdom, rather than the animal kingdom. In addition, the existence of natural hybrids among yeasts suggests that horizontal transfer of genetic material is a significant additional source of genetic variation within the fungal kingdom.

\section{ACKNOWLEDGEMENTS}

Part of this work was supported by grants from the Danish Research Council and the Carlsberg Foundation. C.G. acknowledges a stipend from the Leo Foundation. The authors would like to thank Torsten Nilsson-Tillgren, Michael L. Nielsen and Randi F. Petersen for their interest in this work.

\section{REFERENCES}

Barnett, J. A. (1992). The taxonomy of the genus Saccharomyces Meyen ex Reess: a short review for non-taxonomists. Yeast $\mathbf{8}$, $1-23$.

Felsenstein, J. (1989). PHYLIP - phylogeny inference package (version 3.2). Cladistics 5, 164-166.

Goyon, C., Faugeron, G. \& Rossignol, J. L. (1988). Molecular cloning and characterization of the met 2 gene from Ascobolus immersus. Gene 63, 297-308.

Groth, C. (1998). Saccharomyces sensu stricto yeasts: characterization of mitochondrial DNA. MSc thesis. University of Copenhagen, Copenhagen, Denmark.

Hansen, J. \& Kielland-Brandt, M. C. (1994). Saccharomyces carlsbergensis contains two functional MET2 alleles similar to homologues from $S$. cerevisiae and S. monacensis. Gene $\mathbf{1 4 0}$, $33-40$.

Hoffman, C. S. \& Winston, F. (1987). A ten-minute DNA preparation from yeast efficiently releases autonomous plasmids for transformation of Escherichia coli. Gene 57, 267-272.

Kaneko, Y. \& Banno, I. (1991). Re-examination of Saccharomyces bayanus strains by DNA-DNA hybridization and electrophoretic karyotyping. Inst Ferm Res Comm (Osaka) 15, 30-41.

Kielland-Brandt, M., Nilsson-Tillgren, T., Gjermansen, C., Holmberg, S. \& Pedersen, M. B. (1995). In The Yeasts, vol. 6, pp. 223-254. Edited by A. H. Rose, A. E. Wheals \& J. S. Harrison. London: Academic Press.

Langin, T., Faugeron, G., Goyon, C., Nicolas, A. \& Rossignol, J. L. (1986). The MET2 gene of Saccharomyces cerevisiae: molecular cloning and nucleotide sequence. Gene 49, 283-293.

Li, M., Tzagoloff, A., Underbrink-Lyon, K. \& Martin, N. C. (1982). Identification of the paromomycin-resistance mutation in the
15S rRNA gene of yeast mitochondria. J Biol Chem 257, 5921-5928.

Macreadie, I. G., Novitski, C. E., Maxwell, R. J., John, U., Ooi, B. G., McMullen, G. L., Lukins, H. B., Linnane, A. W. \& Nagley, P. (1983). Biogenesis of mitochondria: the mitochondrial gene (aapl) coding for mitochondrial ATPase subunit 8 in Saccharomyces cerevisiae. Nucleic Acids Res 11, 4435-4451.

Masneuf, I., Hansen, J., Groth, C., Piškur, J. \& Dubourdieu, D. (1998). New hybrids between Saccharomyces sensu stricto yeast species found among wine and cider production strains. Appl Environ Microbiol 64, 3887-3892.

Naumov, G. I. (1996). Genetic identification of biological species in the Saccharomyces sensu stricto complex. J Ind Microbiol 17, 295-302.

Naumov, G. I., Naumova, E. S., Hagler, A. N., Mendonça-Hagler, L. C. \& Louis, E. J. (1995a). A new genetically isolated population of the Saccharomyces sensu stricto complex from Brazil. Antonie Leeuwenhoek 67, 351--355.

Naumov, G. I., Naumova, E. S. \& Louis, E. J. (1995b). Two new genetically isolated populations of the Saccharomyces sensu stricto complex from Japan. J Gen Appl Microbiol 41, 499-505.

Nicoletti, L., Laveder, R., Pellizzari, R., Cardazzo, B. \& Carignani, G. (1994). Comparative analysis of the region of the mitochondrial genome containing the ATPase subunit 9 gene in the two related yeast species Saccharomyces douglasii and Saccharomyces cerevisiae. Curr Genet 25, 504-507.

Ooi, B. G., McMullen, G. L., Linnane, A. W., Nagley, P. \& Novitski, C. E. (1985). Biogenesis of mitochondria: DNA sequence analysis of mit ${ }^{-}$mutations in the mitochondrial olil gene coding for mitochondrial ATPase subunit 9 in Saccharomyces cerevisiae. Nucleic Acids Res 13, 1327-1339.

Page, R. D. (1996). TreeView: an application to display phylogenetic trees on personal computers. Comput Appl Biosci 12, 357-358.

Pedersen, M. B. (1986). DNA sequence polymorphisms in the genus Saccharomyces. IV. Homologous chromosomes III in Saccharomyces bayanus, $S$. carlsbergensis, and $S$. uvarum. Carlsberg Res Commun 51, 185-202.

Pǐ̌kur, J. (1989). Respiratory-competent yeast mitochondrial DNAs generated by deleting intergenic regions. Gene $\mathbf{8 1}$, 165-168.

PiY̌kur, J., Smole, S., Groth, C., Petersen, R. F. \& Pedersen, M. B. (1998). Structure and genetic stability of mitochondrial genomes vary among yeasts of the genus Saccharomyces. Int J Syst Bacteriol 48, 1015-1024.

Sekito, T., Okamoto, K., Kitano, H. \& Yoshida, K. (1995). The complete mitochondrial DNA sequence of Hansenula wingei reveals new characteristics of yeast mitochondria. Curr Genet 28, 39-53.

Thompson, J. D., Higgins, D. G. \& Gibson, T. J. (1994). CLUSTAL $\mathrm{W}$ : improving the sensitivity of progressive multiple sequence alignment through sequence weighting, position-specific gap penalties and weight matrix choice. Nucleic Acids Res 22, 4673-4680. 